\title{
POSET LIMITS CAN BE TOTALLY ORDERED
}

\author{
JAN HLADKÝ, ANDRÁS MÁTHÉ, VIRESH PATEL, AND OLEG PIKHURKO
}

\begin{abstract}
S. Janson [Poset limits and exchangeable random posets, Combinatorica 31 (2011), 529-563] defined limits of finite posets in parallel to the emerging theory of limits of dense graphs.

We prove that each poset limit can be represented as a kernel on the unit interval with the standard order, thus answering an open question of Janson. We provide two proofs: real-analytic and combinatorial. The combinatorial proof is based on a Szemerédi-type Regularity Lemma for posets which may be of independent interest.

Also, as a by-product of the analytic proof, we show that every atomless ordered probability space admits a measure-preserving and almost orderpreserving map to the unit interval.
\end{abstract}

\section{INTRODUCTION}

Given a class $\mathcal{C}$ of finite structures and some measure $t(F, G)$ for $F, G \in \mathcal{C}$ of how frequently $F$ appears in $G$ as a substructure, one can say that a sequence $\left\{G_{n}\right\}_{n \in \mathbb{N}}$ converges if $\left\{t\left(F, G_{n}\right)\right\}_{n \in \mathbb{N}}$ converges for every $F \in \mathcal{C}$.

For example, if $\mathcal{C}$ consists of finite graphs and $t$ denotes the subgraph density, then we obtain the convergence of (dense) graphs whose systematic study was initiated by Lovász and Szegedy [13] and Borgs et al. [4]. In particular, Lovász and Szegedy [13] showed that for every convergent sequence $\left\{G_{n}\right\}_{n \in \mathbb{N}}$ of graphs there is a measurable function $W:[0,1]^{2} \rightarrow[0,1]$ (called graphon) such that for every graph $F$ the limit of $t\left(F, G_{n}\right)$ as $n \rightarrow \infty$ is a certain integral involving $W$. In fact, many other parameters of $G_{n}$ can be well approximated as $n \rightarrow \infty$ if we know $W$. This opens a general way of bringing analytic methods into the study of large finite graphs. Connections to other areas are established by alternative representations of "graph limits": by reflection positive graph parameters (Lovász and Szegedy [13]), by positive flag algebra homomorphisms (Razborov [17]), and by partially exchangeable random arrays (Diaconis and Janson [6]).

The theory of graph limits has received a great deal of attention and has been extended to other structures as well, such as hypergraphs (Elek and Szegedy [8],

Received by the editors November 11, 2012 and, in revised form, August 2, 2013.

2010 Mathematics Subject Classification. Primary 06A06, 28Axx.

Key words and phrases. Homomorphism density, ordered probability space, partially ordered set, poset kernel, Regularity Lemma.

The first author was supported by an EPSRC fellowship.

The second author was supported by the EPSRC (grant EP/G050678/1), the Hungarian Scientific Research Fund (grants 72655 and 104178) and the Leverhulme Trust.

The third author was supported by the EPSRC (grant EP/J008087/1).

The fourth author was supported by the European Research Council (grant agreement no. 306493) and the National Science Foundation of the USA (grant DMS-1100215). 
see also Tao [21 and Austin [1]), permutations (Hoppen et al. 9, 10]), functions on compact Abelian groups (Szegedy [19]), and others.

An analogous theory for limits of posets (i.e. partially ordered sets) was initiated by Brightwell and Georgiou [5] and further developed by Janson [11]. Let us state some of these results.

We represent a poset as a pair $(P, \prec)$ where $P$ is a finite ground set and $\prec$ is a strict order relation (i.e. it is transitive and no $a \in P$ satisfies $a \prec a$ ).

A map $f: P \rightarrow Q$ (not necessarily injective) is a homomorphism from $(P, \prec)$ to $(Q, \ll)$ if we have $f(x) \ll f(y)$ for every $x, y \in P$ with $x \prec y$. The density $t((P, \prec),(Q, \ll))$ is the number of homomorphisms from $(P, \prec)$ to $(Q, \ll)$ divided by the total number of possible maps $P \rightarrow Q$. In other words, it is the probability that a random map $P \rightarrow Q$ between the ground sets preserves the order relation.

Definition 1.1. A sequence of posets $\left\{\left(P_{n}, \prec_{n}\right)\right\}_{n \in \mathbb{N}}$ converges if $\left|P_{n}\right| \rightarrow \infty$ and

$$
\left\{t\left((P, \prec),\left(P_{n}, \prec_{n}\right)\right)\right\}_{n \in \mathbb{N}} \text { converges for every poset }(P, \prec) \text {. }
$$

Remark 1.2. It is not hard to show (cf. [13, Section 2.4]) that Definition 1.1]does not change if we modify $t$ to be the density of induced and/or injective homomorphisms.

The potential usefulness of (10) comes from the result of Janson 11, Theorem 1.7] that for each convergent sequence there is an analytic limit object as follows. (See Section 2 for an overview of the measure theory notation that we use.)

Definition 1.3. An ordered probability space $(S, \mathcal{F}, \mu, \triangleleft)$ is a probability space $(S, \mathcal{F}, \mu)$ equipped with a strict order relation $\triangleleft$ such that $\{(x, y): x \triangleleft y\}$ is an $\mathcal{F} \otimes \mathcal{F}$-measurable subset of $S \times S$.

Definition 1.4. A (poset) kernel is a 5-tuple $(S, \mathcal{F}, \mu, \triangleleft, W)$, where $(S, \mathcal{F}, \mu, \triangleleft)$ is an ordered probability space and $W$ is an $\mathcal{F} \otimes \mathcal{F}$-measurable function $S \times S \rightarrow[0,1]$ such that, for all $x, y, z \in S$,

$$
\begin{aligned}
W(x, y)>0 & \Rightarrow x \triangleleft y, \\
W(x, y)>0 \text { and } W(y, z)>0 & \Rightarrow W(x, z)=1 .
\end{aligned}
$$

In particular, it follows from Definition 1.4 that $W(x, y) W(y, x)=0$ for every $x, y \in S$.

When no confusion arises, we may abbreviate $(P, \prec)$ to $P$ and $(S, \mathcal{F}, \mu, \triangleleft, W)$ to $W$. Also, we will usually say "kernel" instead of "poset kernel".

Definition 1.5. The density of a poset $(P, \prec)$ in a kernel $(S, \mathcal{F}, \mu, \triangleleft, W)$ is

$$
t(P, W):=\int_{S|P|} \prod_{\substack{a, b \in P \\ a \prec b}} W\left(x_{a}, x_{b}\right) \prod_{a \in P} \mathrm{~d} \mu\left(x_{a}\right) .
$$

There is some analogy between $t(P, Q)$ and $t(P, W)$. Namely, one can interpret the expression in the right-hand side of (4) as follows. Select random elements $x_{a}$ of $(S, \mathcal{F}, \mu)$ indexed by $P$ and let $x_{a} \ll x_{b}$ with probability $W\left(x_{a}, x_{b}\right)$, with all choices being mutually independent. Then $t(P, W)$ is exactly the probability that $x_{a} \ll x_{b}$ for all $a \prec b$ in $P$. In fact, the connection is much deeper as the following result shows. 
Theorem 1.6 (Janson [11, Theorems 1.7 and 1.9(ii)]). For every convergent sequence $\left\{P_{n}\right\}_{n \in \mathbb{N}}$ of posets there is a kernel $(S, \mathcal{F}, \mu, \triangleleft, U)$ such that

$$
t(P, U)=\lim _{n \rightarrow \infty} t\left(P, P_{n}\right), \text { for every poset } P .
$$

Moreover, we can assume in (5) that

$$
(S, \mathcal{F}, \mu)=([0,1], \mathcal{B}, \lambda)
$$

is the unit interval with the Lebesgue measure $\lambda$ on the Borel $\sigma$-algebra $\mathcal{B}$.

Also, the converse of Theorem [1.6 was established in [1]: for every kernel $U$ there is a sequence of posets $\left\{P_{n}\right\}_{n \in \mathbb{N}}$ that satisfies (5). In fact, the sampling procedure informally described after (4) yields such a sequence with probability 1.

Although we can require that (6) holds, the proof in [11] gives no control over the order relation $\triangleleft$. This prompted Janson [11, Problem 1.10] to ask if one can always take $([0,1], \mathcal{B}, \lambda,<)$ with the standard order $<$ in Theorem 1.6. In a later paper [12, Janson answered his question for convergent sequences of interval orders (see also [11, Theorem 1.9(iii)] for a related result). Here we give the affirmative answer in the general case.

Theorem 1.7. For every convergent sequence $\left\{P_{n}\right\}_{n \in \mathbb{N}}$ of posets there is a kernel $([0,1], \mathcal{B}, \lambda,<, U)$ such that (5) holds.

In fact, we provide two different proofs of Theorem 1.7

One goes via a Regularity Lemma for posets that we prove in Section 6. Our lemma finds a partition $P=V_{1} \cup \cdots \cup V_{k}$ which is $\varepsilon$-regular with respect to the underlying graph of $(P, \prec)$ and has the additional property that all $\prec$-relations between parts go "forward" only. Having such a Regularity Lemma, we follow the method of Lovász and Szegedy [13,14] to construct a kernel $U$ by taking the "limit" of $\varepsilon$-regular partitions as $\varepsilon \rightarrow \infty$. The above "forward" property allows us to ensure that $U(x, y)=0$ whenever $x \geq y$, thus proving Theorem 1.7. We expect our Regularity Lemma to have further applications.

The other proof of Theorem 1.7 is real-analytic. Actually, we prove a somewhat stronger result (Theorem 1.9 below). In order to state it, we have to give some further definitions.

Let $(S, \mathcal{F}, \mu, \triangleleft, W)$ be a kernel. We call it strict if $W(x, y)>0$ for every $x, y \in S$ with $x \triangleleft y$. (Thus a kernel is strict if the two sides of (2) are equivalent.) Kernel axioms imply that if we define $\triangleleft^{\prime}:=\left\{(x, y) \in S^{2}: W(x, y)>0\right\}$, then $\left(S, \mathcal{F}, \mu, \triangleleft^{\prime}\right)$ is an ordered probability space on which $W$ is a strict kernel. Clearly, this change does not affect (4). Thus, we can addionally assume in Theorem 1.6 that $U$ is strict; see [11, Remark 1.2].

Definition 1.8. An inclusion between ordered probability spaces $(S, \mathcal{F}, \mu, \triangleleft)$ and $\left(S^{\prime}, \mathcal{F}^{\prime}, \mu^{\prime}, \triangleleft^{\prime}\right)$ is a measure-preserving function $f: S \rightarrow S^{\prime}$ such that the set

$$
\left\{(x, y) \in S^{2}: x \triangleleft y, f(x) 丸^{\prime} f(y)\right\}
$$

has $\mu \otimes \mu$-measure zero. Additionally, if we have a kernel $U$ on $\left(S^{\prime}, \mathcal{F}^{\prime}, \mu^{\prime}, \triangleleft^{\prime}\right)$, then its pull-back along $f$ is the function $U^{f}: S^{2} \rightarrow[0,1]$, defined by $U^{f}(x, y):=$ $U(f(x), f(y))$ for $x, y \in S$.

Theorem 1.9. For every strict kernel $(S, \mathcal{F}, \mu, \triangleleft, W)$ such that $(S, \mathcal{F}, \mu)$ is atomless, there is a kernel $([0,1], \mathcal{B}, \lambda,<, U)$ and an inclusion $f:(S, \mathcal{F}, \mu, \triangleleft) \rightarrow$ $([0,1], \mathcal{B}, \lambda,<)$ such that $W$ is equal to the pull-back $U^{f}$ almost everywhere. 
Since $f$ in Theorem 1.9 is measure-preserving, we necessarily have that $t(P, U)=$ $t(P, W)$ for every poset $P$, that is, $U$ and $W$ represent the same poset limit. Thus Theorem 1.7 follows from Theorems 1.6 and 1.9 .

The notion of a pull-back plays an important role in the theory of graphons. Hopefully, our Theorem [1.9 will be generally useful when studying poset kernels. For example, if the studied kernel property behaves well with respect to taking pull-backs, then one can operate with the function $U$ that satisfies Theorem 1.9 instead of the 5 -tuple $(S, \mathcal{F}, \mu, \triangleleft, W)$.

Given an ordered probability space $(S, \mathcal{F}, \mu, \triangleleft)$, the indicator function $I_{\triangleleft}$ of the order relation $\triangleleft$ is clearly a strict kernel on it. Thus Theorem 1.9 has the following direct corollary.

Theorem 1.10. Every atomless ordered probability space $(S, \mathcal{F}, \mu, \triangleleft)$ can be included into $([0,1], \mathcal{B}, \lambda,<)$.

Theorem 1.10 can be viewed as a measure theoretic analogue of the statement that every poset can be totally ordered. While extending this to infinite partially ordered sets is an easy application of Zorn's lemma, the main content of Theorem 1.10 is that this total ordering can be done in a "measurable" way. Interestingly, the limit of totally ordered increasing posets happens to be our universal target space $([0,1], \mathcal{B}, \lambda,<)$ with the indicator function $I_{<}$as its kernel.

This paper is organised as follows. Section 2 describes the measure theory notation that we frequently use. Section 3 presents some auxiliary analytic lemmas, thus making the flow of arguments in the later sections smoother. Although Theorem 1.10 is a direct consequence of Theorem 1.9. we prove it first in Section 4. Then, in Section 5, we show how Theorem 4.1 (a version of Theorem 1.10) implies Theorem 1.9. Our Regularity Lemma for posets is stated and proved in (combinatorial) Section 6 which can be read independently of the other sections. We show how this Regularity Lemma gives an alternative proof of Theorem 1.7 in Section 7 Finally, Section 8 contains some concluding remarks, including examples that certain strengthenings of our results are not possible.

\section{MeAsure theORY NOtATion}

Let us give some notation that we are going to use frequently. We do not define many standard concepts of measure theory but refer the reader to Bogachev's book [2] whose notation we generally follow. We try to provide sufficient references and explanations so that this paper is accessible to combinatorialists who do not have a strong background in measure theory.

Let $\mathbb{N}=\{1,2, \ldots\}$ and $\mathbb{R}$ be the sets of respectively natural and real numbers. When we consider a subset of $\mathbb{R}$, typically the unit interval $[0,1]$, we will denote the $\sigma$-algebra of its Borel subsets by $\mathcal{B}$ and the Lebesgue measure by $\lambda$. For a family $\mathcal{X}$ of sets, let $\sigma(\mathcal{X})$ denote the $\sigma$-algebra generated by $\mathcal{X}$. Let $I_{X}$ denote the indicator function of a set $X$ (that is, $I_{X}(x)$ is 1 if $x \in X$ and 0 otherwise).

Let $(S, \mathcal{F}, \mu)$ be a probability space. As it is standard in measure theory, a realvalued function $f$ on $S$ is called $\mathcal{F}$-measurable if it is $(\mathcal{F}, \mathcal{B})$-measurable. We denote by $\mathcal{F}_{\mu}$ the completion of $\mathcal{F}$ with respect to the measure $\mu$.

We say that a property holds $(\mathcal{F}, \mu)$-almost everywhere (and abbreviate this to $(\mathcal{F}, \mu)$-a.e. $)$ if the set of points of $S$ where it fails belongs to $\mathcal{F}_{\mu}$ and has $\mu$-measure zero. When the underlying measure space is understood, we just write "a.e." In 
some rare cases when we consider more than one $\sigma$-algebra on the same set, the bare term "a.e." refers to the largest $\sigma$-algebra.

We call two sets or two functions equivalent (and use the symbol $\sim$ ) if they coincide a.e. The Fréchet-Nikodym distance between two sets $A, B \in \mathcal{F}_{\mu}$ is $\mu(A \triangle B)$; it is in general a pseudo-metric (it satisfies the Triangle Inequality but may evaluate to 0 for $A \neq B)$. The space $(S, \mathcal{F}, \mu)$ is called separable if $\mathcal{F}$ has a countable subset which is dense with respect to the Fréchet-Nikodym distance.

Let $\mathcal{A} \subseteq \mathcal{F}$ be another $\sigma$-algebra and let $f$ be an integrable real-valued function on $(S, \mathcal{F}, \mu)$. The conditional expectation $\mathbb{E}(f \mid \mathcal{A})$ is the set of all $\mathcal{A}$-measurable functions $g: S \rightarrow \mathbb{R}$ such that for every bounded $\mathcal{A}$-measurable function $h: S \rightarrow \mathbb{R}$ we have

$$
\int h(x) g(x) \mathrm{d} \mu(x)=\int h(x) f(x) \mathrm{d} \mu(x) .
$$

As is well known, $\mathbb{E}(f \mid \mathcal{A}) \neq \emptyset$ and every two functions in $\mathbb{E}(f \mid \mathcal{A})$ are equivalent; also, it is enough to check (8) for $\{0,1\}$-valued $h$ only, i.e. that

$$
\int_{A} g(x) \mathrm{d} \mu(x)=\int_{A} f(x) \mathrm{d} \mu(x), \quad \text { for all } A \in \mathcal{A} .
$$

We refer the reader to [2, Section 10.1.1] for some basic properties of conditional expectation. We may treat $\mathbb{E}(f \mid \mathcal{A})$ as a single function (rather as a set of functions), when the studied property does not depend on the choice of a representative.

Let $\left(S^{\prime}, \mathcal{F}^{\prime}, \mu^{\prime}\right)$ be another probability space. A map $f: S \rightarrow S^{\prime}$ is measurepreserving if $f$ is $\left(\mathcal{F}, \mathcal{F}^{\prime}\right)$-measurable and, for every $A \in \mathcal{F}^{\prime}$, we have $\mu\left(f^{-1}(A)\right)=$ $\mu^{\prime}(A)$. The products of $\sigma$-algebras and measures are denoted by $\mathcal{F} \otimes \mathcal{F}^{\prime}$ and $\mu \otimes \mu^{\prime}$. We use the shorthand $\mathcal{F} \otimes \mathcal{F}^{\prime}$ for $\left(\mathcal{F} \otimes \mathcal{F}^{\prime}\right)_{\mu \otimes \mu^{\prime}}$, the completion of $\mathcal{F} \otimes \mathcal{F}^{\prime}$ with respect to $\mu \otimes \mu^{\prime}$. We will be using Fubini's theorem ([2, Theorem 3.4.4]) very frequently, often without explicitly mentioning it. Let us stress that one has to be careful when dealing with products of $\sigma$-algebras and measures. For example, the product of two complete measure spaces is not complete in general. Also, see Exercises 44-45, 49-51, and 55 in [2, Section 3.10] for counterexamples to some "plausible" statements related to Fubini's theorem.

Let us give some kernel-specific definitions (when the underlying ordered probability space $(S, \mathcal{F}, \mu, \triangleleft)$ is understood). For $A \subseteq S$, let $A^{c}:=S \backslash A$ denote the complement of $A$. For $X \in \mathcal{F} \otimes \mathcal{F}$, we define

$$
\mu_{\triangleleft}(X):=\int_{X} I_{\triangleleft}(a, b) \mathrm{d} \mu(a) \mathrm{d} \mu(b) .
$$

For a 2 -variable function $W: S^{2} \rightarrow \mathbb{R}$ and $y \in S$, the slice function $W_{y}: S \rightarrow \mathbb{R}$ is defined by $W_{y}(x):=W(x, y)$. We call $W: S \times S \rightarrow[0,1]$ an almost (poset) kernel if $W$ is $\mathcal{F} \otimes \mathcal{F}$-measurable and the kernel axioms (2) and (3) hold for a.e. triple $(x, y, z)$ in $(S, \mathcal{F}, \mu)^{3}$.

Although our Theorem 1.9 takes a kernel $W$ as input and then produces another kernel $U$, we have to deal with almost kernels at intermediate stages of the proof. (For example, the pull-back $U^{f}$ in Theorem 1.9 is generally an almost kernel.)

\section{Auxiliary analytic lemmas}

Here we present some auxiliary results that we will need later.

Janson [11] proved that one can transform an almost kernel $(S, \mathcal{B}, \lambda, \triangleleft, W)$ with $S \subseteq \mathbb{R}$ into a kernel $\left(S, \mathcal{B}, \lambda, \triangleleft^{\prime}, W^{\prime}\right)$ with $W^{\prime} \sim W$ and some $\triangleleft^{\prime}$. We show that in 
the special case of the unit interval with the standard order, one can also keep the order relation intact.

Lemma 3.1. Let $([0,1], \mathcal{B}, \lambda,<, U)$ be an almost kernel. Then there is $U^{\prime} \sim U$ such that $\left([0,1], \mathcal{B}, \lambda,<, U^{\prime}\right)$ is a kernel.

Proof. First, we choose a $\mathcal{B} \otimes \mathcal{B}$-measurable function $U_{0} \sim U$; it exists by [2, Proposition 2.1.11]. Then we proceed in a similar fashion as is done by Janson [11, pages 547-548], so we will be rather brief. We refer the reader to [2, Section 5] for the definitions and basic properties of Lebesgue and density points. Here, these are defined relative to the domain of a function; namely, the system of shrinking neighbourhoods around $(x, y) \in[0,1]^{2}$ is taken to be $(x \pm \varepsilon) \times(y \pm \varepsilon)$ as $\varepsilon \rightarrow 0$, where e.g. $(x \pm \varepsilon)$ denotes the intersection of the open interval $(x-\varepsilon, x+\varepsilon)$ with $[0,1]$.

We define $U_{1}:[0,1]^{2} \rightarrow[0,1]$ by $U_{1}(x, y):=U_{0}(x, y)$ if $(x, y) \in[0,1]^{2}$ is a Lebesgue point of $U_{0}$. Next, if $(x, y)$ is a density point of the set $\left\{(x, y): U_{0}(x, y)=\right.$ $1\}$, then let $U_{1}(x, y):=1$. (Recall that a density point need not belong to the set itself.) For all other pairs $(x, y) \in[0,1]^{2}$, we define $U_{1}(x, y):=0$. Note that $U_{1} \sim U_{0}$ and $U_{1}$ is still $\mathcal{B} \otimes \mathcal{B}$-measurable.

We claim that $U_{1}$ is a kernel on $([0,1], \mathcal{B}, \lambda,<)$. Suppose that $U_{1}(x, y)>0$. Then for every sufficiently small $\varepsilon>0$, we have $U_{0}\left(x^{\prime}, y^{\prime}\right)>0$ for most points $\left(x^{\prime}, y^{\prime}\right) \in(x \pm \varepsilon) \times(y \pm \varepsilon)$. In particular, $x^{\prime}<y^{\prime}$ for most of these pairs and therefore $x<y$.

Now suppose that $U_{1}(x, y)>0$ and $U_{1}(y, z)>0$. Then for every sufficiently small $\varepsilon>0$, we have $U_{0}\left(x^{\prime}, y^{\prime}\right)>0$ for most points $\left(x^{\prime}, y^{\prime}\right) \in(x \pm \varepsilon) \times(y \pm \varepsilon)$ and $U_{0}\left(y^{\prime}, z^{\prime}\right)>0$ for most points $\left(y^{\prime}, z^{\prime}\right) \in(y \pm \varepsilon) \times(z \pm \varepsilon)$. This implies that we have $U_{0}\left(x^{\prime}, z^{\prime}\right)=1$ for most points $\left(x^{\prime}, z^{\prime}\right) \in(x \pm \varepsilon) \times(z \pm \varepsilon)$. Thus $(x, z)$ is a density point of $\left\{(x, y): U_{0}(x, y)=1\right\}$ and therefore $U_{1}(x, z)=1$, as required.

Lemma 3.2 (Borgs, Chayes, and Lovász 3. Lemma 3.4]). Let $(S, \mathcal{F})$ and $\left(S^{\prime}, \mathcal{F}^{\prime}\right)$ be measurable spaces, and let $W: S \times S^{\prime} \rightarrow \mathbb{R}$ be a bounded $\mathcal{F} \otimes \mathcal{F}^{\prime}$-measurable function. Then there exist countably generated $\sigma$-algebras $\mathcal{F}_{0} \subseteq \mathcal{F}$ and $\mathcal{F}_{0}^{\prime} \subseteq \mathcal{F}^{\prime}$ such that $W$ is $\mathcal{F}_{0} \otimes \mathcal{F}_{0}^{\prime}$-measurable.

Lemma 3.3. Let $f: S \rightarrow S^{\prime}$ be an inclusion of ordered probability spaces $(S, \mathcal{F}, \mu, \triangleleft)$ and $\left(S^{\prime}, \mathcal{F}^{\prime}, \mu^{\prime}, \triangleleft^{\prime}\right)$. Let $W$ be a kernel on $S$ such that $W \sim \mathbb{E}(W \mid \mathcal{A} \otimes \mathcal{A})$, where $\mathcal{A}:=f^{-1}\left(\mathcal{F}^{\prime}\right)$. Then there is an almost kernel $U$ on $\left(S^{\prime}, \mathcal{F}^{\prime}, \mu^{\prime}, \triangleleft^{\prime}\right)$ with $W \sim U^{f}$.

Proof. We construct $U$ following the argument of Borgs, Chayes, and Lovász [3, Lemma 3.1].

Note that $f \times f:(S, \mathcal{F}, \mu)^{2} \rightarrow\left(S^{\prime}, \mathcal{F}^{\prime}, \mu^{\prime}\right)^{2}$ is measure-preserving. Define a measure $\nu$ on $\mathcal{F}^{\prime} \otimes \mathcal{F}^{\prime}$ by

$$
\nu(X):=\int_{(f \times f)^{-1}(X)} W(x, y) \mathrm{d} \mu(x) \mathrm{d} \mu(y), \quad X \in \mathcal{F}^{\prime} \otimes \mathcal{F}^{\prime} .
$$

This measure $\nu$ is absolutely continuous with respect to $\mu^{\prime} \otimes \mu^{\prime}$. Hence, the RadonNikodym derivative

$$
U:=\frac{\mathrm{d} \nu}{\mathrm{d}\left(\mu^{\prime} \otimes \mu^{\prime}\right)}
$$

exists ([2, Theorem 3.2.2]). Namely, $U: S^{\prime} \times S^{\prime} \rightarrow \mathbb{R}$ is a $\mu^{\prime} \otimes \mu^{\prime}$-integrable function such that for every $X \in \mathcal{F}^{\prime} \otimes \mathcal{F}^{\prime}$ we have $\nu(X)=\int_{X} U \mathrm{~d}\left(\mu^{\prime} \otimes \mu^{\prime}\right)$. 
The last identity implies (given that $f$ is measure-preserving and that $0 \leq W \leq$ 1) that the set

$$
\left\{(x, y) \in S^{\prime} \times S^{\prime}: U(x, y)>1 \text { or } U(x, y)<0\right\}
$$

has measure zero. By changing $U$ on a null set, we can assume that $U$ is $\mathcal{F}^{\prime} \otimes \mathcal{F}^{\prime}$ measurable (see [2, Proposition 2.1.11]) and that the values of $U$ belong to [0,1]. In particular, the pull-back $U^{f}$ is $\mathcal{A} \otimes \mathcal{A}$-measurable. Moreover, for any $Y \in \mathcal{A} \otimes \mathcal{A}$, say $Y=(f \times f)^{-1}(X)$, we have that

$$
\int_{Y} U^{f} \mathrm{~d}(\mu \otimes \mu)=\int_{X} U \mathrm{~d}\left(\mu^{\prime} \otimes \mu^{\prime}\right)=\int_{Y} W \mathrm{~d}(\mu \otimes \mu) .
$$

By (9), we conclude that $U^{f} \in \mathbb{E}(W \mid \mathcal{A} \otimes \mathcal{A})$. Thus $U^{f}$ is a.e. equal to $W$ by the assumption of the lemma.

Let us verify that $U$ is an almost kernel. First, consider the set

$$
X:=\left\{(x, y) \in S^{\prime} \times S^{\prime}: x \not^{\prime} y, U(x, y)>0\right\} \in \mathcal{F}^{\prime} \otimes \mathcal{F}^{\prime}
$$

of points where the first kernel axiom (2) fails for $U$. By (11), the integral of $U$ over $X$ is the same as the integral of $W$ over $Y:=(f \times f)^{-1}(X)$. Since $f$ is an inclusion, we have $\mu_{\triangleleft}(Y)=0$, where $\mu_{\triangleleft}$ is defined by (10). Since $W$ is a kernel, it is zero a.e. on $Y$. It follows that $X$ has measure zero, that is, $U$ satisfies (2) a.e.

Define $u(x, y, z):=U(x, y) U(y, z)(1-U(x, z))$. Since $U^{f} \sim W$ and $W$ is a kernel, we have $u^{f} \sim 0$. Since $f$ is measure-preserving, we have $\int u=\int u^{f}=0$. The non-negativity of $u$ implies that $u \sim 0$, that is, $U$ satisfies (3) a.e.

Remark 3.4. The conditional expectation of a kernel need not be an almost kernel. For example, let $S:=\{a, b, c, d\}$ with $\mathcal{F}:=2^{S}$ and $\mu$ being the uniform measure. Let $\mathcal{A}=\sigma(\{a\},\{b, c\},\{d\}) \subseteq \mathcal{F}$ be obtained by "gluing" $b$ and $c$ together. Let $a \triangleleft b$ and $c \triangleleft d$ be all order relations and let $W:=I_{\triangleleft}$. Then any $U \in \mathbb{E}(W \mid \mathcal{A} \otimes \mathcal{A})$ satisfies $U(a, b)=1 / 2, U(b, d)=1 / 2$, and $U(a, d)=0$ and cannot be an almost kernel. Also, pull-backs do not preserve (almost) kernels in general: for example, the pull-back of $I_{<}$with respect to the identity inclusion of $([0,1], \mathcal{B}, \lambda, \emptyset)$ into $([0,1], \mathcal{B}, \lambda,<)$ does not satisfy (2) .

Lemma 3.5. Let $(S, \mathcal{F}, \mu)$ be a probability space. Let $\mathcal{A} \subseteq \mathcal{F}$ be another $\sigma$-algebra such that $(S, \mathcal{A}, \mu)$ is separable. Let $W: S^{2} \rightarrow \mathbb{R}$ be a bounded $\mathcal{F} \otimes \mathcal{F}$-measurable function. Let $g \in \mathbb{E}(W \mid \mathcal{A} \otimes \mathcal{F})$. Then, for a.e. $y \in S$, we have that $g_{y} \sim \mathbb{E}\left(W_{y} \mid \mathcal{A}\right)$.

Proof. By definition, $g$ is $\mathcal{A} \otimes \mathcal{F}$-measurable. It follows by [2, Proposition 3.3.2] that the slice function $g_{y}$ is $\mathcal{A}$-measurable for every $y \in S$.

Fix $A \in \mathcal{A}$. By the definition of conditional expectation, we have that $\int_{A \times B} g=$ $\int_{A \times B} W$ for every $B \in \mathcal{F}$. Likewise,

$$
\int_{A} \mathbb{E}\left(W_{y} \mid \mathcal{A}\right)=\int_{A} W_{y}, \quad \text { for every } y \in S .
$$

By Fubini's theorem, the latter function is integrable as a function of $y$. Moreover,

$$
\int_{B}\left(\int_{A} W_{y}(x) \mathrm{d} \mu(x)\right) \mathrm{d} \mu(y)=\int_{A \times B} W=\int_{A \times B} g=\int_{B}\left(\int_{A} g_{y}(x) \mathrm{d} \mu(x)\right) \mathrm{d} \mu(y) .
$$


Since $B \in \mathcal{F}$ was arbitrary, [2, Corollary 2.5.4] gives that $\int_{A} W_{y}=\int_{A} g_{y}$ for a.e. $y$. Let us remove all exceptional points $y$ when $A$ runs over a dense countable subset $\left\{A_{1}, A_{2}, \ldots\right\} \subseteq \mathcal{A}$ in $(S, \mathcal{A}, \mu)$ as well as those $y$ for which $\left\|g_{y}\right\|_{\infty}>\|W\|_{\infty}$ or $\left\|W_{y}\right\|_{\infty}>\|W\|_{\infty}$. It is easy to see that the remaining set $Y$ has measure 1.

Fix any $y \in Y$. For every $A \in \mathcal{A}$ we have that

$$
\left|\int_{A} W_{y}-\int_{A} g_{y}\right| \leq\left|\int_{A} W_{y}-\int_{A_{i}} W_{y}\right|+\left|\int_{A} g_{y}-\int_{A_{i}} g_{y}\right| \leq 4\|W\|_{\infty} \mu\left(A \triangle A_{i}\right) .
$$

Since the right-hand side can be made arbitrarily small by choosing a suitable $A_{i}$, we conclude that $\int_{A} W_{y}=\int_{A} g_{y}$. Since $A \in \mathcal{A}$ was arbitrary and both $\mathbb{E}\left(W_{y} \mid \mathcal{A}\right)$ and $g_{y}$ are $\mathcal{A}$-measurable, they coincide a.e. by (12). The lemma is proved as $\mu(S \backslash Y)=0$.

\section{Proof of Theorem 1.10}

Let $(S, \mathcal{F}, \mu, \triangleleft)$ be given. Lemma 3.2 , when applied to the indicator function $I_{\triangleleft}$, returns two countably generated $\sigma$-algebras $\mathcal{F}_{0}, \mathcal{F}_{0}^{\prime} \subseteq \mathcal{F}$. Let $\mathcal{F}^{\prime}:=\sigma\left(\mathcal{F}_{0} \cup \mathcal{F}_{0}^{\prime}\right)$ be the $\sigma$-algebra on $S$ generated by $\mathcal{F}_{0} \cup \mathcal{F}_{0}^{\prime}$. By enlarging a set of generators of $\mathcal{F}^{\prime}$ by adding a countably many elements of $\mathcal{F}$, we can additionally make $\left(S, \mathcal{F}^{\prime}, \mu\right)$ atomless.

Clearly, if we prove Theorem 1.10 for this new space $\left(S, \mathcal{F}^{\prime}, \mu, \triangleleft\right)$, then the same inclusion $f$ will work for the original one (as $\mathcal{F}^{\prime} \subseteq \mathcal{F}$ ). Thus, without loss of generality, let us assume that $\mathcal{F}$ is countably generated. It easily follows (see e.g. Exercise 1.12.102 and its hint in [2] that $(S, \mathcal{F}, \mu)$ is separable. Thus it is enough to prove the following theorem (whose last claim will be needed later in Section 5).

Theorem 4.1. Let $(S, \mathcal{F}, \mu, \triangleleft)$ be an ordered probability space such that $(S, \mathcal{F}, \mu)$ is atomless and separable. Then there is an inclusion $f:(S, \mathcal{F}, \mu, \triangleleft) \rightarrow([0,1], \mathcal{B}, \lambda,<)$ such that every set $A \in \mathcal{F}$ with $\mu_{\triangleleft}\left(A \times A^{c}\right)=0$ belongs to $\left(f^{-1}(\mathcal{B})\right)_{\mu}$, the completion of $f^{-1}(\mathcal{B})$ with respect to the measure $\mu$.

So we prove Theorem 4.1 now.

Claim 4.2. Let $B \in \mathcal{F}$ with $\mu(B)>0$. Then there exists $A \in \mathcal{F}$ such that $\mu_{\triangleleft}\left(A \times A^{c}\right)=0, \mu(B \cap A)>0$, and $\mu\left(B \cap A^{c}\right)>0$.

Proof of Claim. Let $\triangleright_{x}:=\{y \in S: y \triangleright x\} \in \mathcal{F}$ be the strict upper shadow of $x \in S$ and let

$$
B^{\prime}:=\left\{x \in B: \mu\left(B \cap \triangleright_{x}\right)>0\right\} .
$$

First, suppose that $\mu\left(B^{\prime}\right)>0$. Clearly, $\mu_{\triangleleft}\left(B^{\prime} \times B^{\prime}\right) \leq \mu\left(B^{\prime}\right)^{2} / 2$. By Fubini's theorem, there is $x \in B^{\prime}$ with $\mu\left(B^{\prime} \cap \triangleright_{x}\right) \leq \mu\left(B^{\prime}\right) / 2$. Clearly, $A:=\triangleright_{x}$ has the required properties.

If $\mu\left(B^{\prime}\right)=0$, then $\mu_{\triangleleft}(B \times B)=0$ by Fubini's theorem. Since $\mathcal{F}$ is atomless, it contains $A^{\prime} \subseteq B$ with $0<\mu\left(A^{\prime} \cap B\right)<\mu(B)$. The function

$$
a(x):=\mu\left(\left\{y \in A^{\prime}: y \triangleleft x\right\}\right)
$$

is $\mathcal{F}$-measurable by [2, Corollary 3.3.3]. The set $X:=\{x \in S: a(x)>0\} \in \mathcal{F}$ is clearly up-closed with respect to $\triangleleft$ and it intersects $B$ in a set of measure 0 by Fubini's theorem. It is easy to see that $A:=A^{\prime} \cup X$ satisfies the claim. 
Let

$$
\mathcal{T}:=\left\{A \in \mathcal{F}: 0<\mu(A)<1, \mu_{\triangleleft}\left(A \times A^{c}\right)=0\right\} .
$$

By Claim 4.2, $\mathcal{T}$ is non-empty and, moreover, infinite. Since $(S, \mathcal{F}, \mu)$ is separable, we can choose a countable subset $\left\{A_{1}, A_{2}, \ldots\right\} \subseteq \mathcal{T}$ which is dense in $\mathcal{T}$ with respect to the Fréchet-Nikodym distance.

We define $f$ so that it satisfies the following properties:

$$
\begin{aligned}
f\left(A_{1}^{c}\right) & \subseteq\left[0, \mu\left(A_{1}^{c}\right)\right], \\
f\left(A_{1}\right) & \subseteq\left[\mu\left(A_{1}^{c}\right), 1\right], \\
f\left(A_{1}^{c} \cap A_{2}^{c}\right) & \subseteq\left[0, \mu\left(A_{1}^{c} \cap A_{2}^{c}\right)\right], \\
f\left(A_{1}^{c} \cap A_{2}\right) & \subseteq\left[\mu\left(A_{1}^{c} \cap A_{2}^{c}\right), \mu\left(A_{1}^{c}\right)\right], \\
f\left(A_{1} \cap A_{2}^{c}\right) & \subseteq\left[\mu\left(A_{1}^{c}\right), \mu\left(A_{1}^{c}\right)+\mu\left(A_{1} \cap A_{2}^{c}\right)\right], \\
f\left(A_{1} \cap A_{2}\right) & \subseteq\left[\mu\left(A_{1}^{c}\right)+\mu\left(A_{1} \cap A_{2}^{c}\right), 1\right],
\end{aligned}
$$

and so on. Specifically, for a (finite or infinite) binary sequence $\mathbf{b}=\left(b_{1}, b_{2}, \ldots\right)$, let

$$
\begin{aligned}
A_{\mathbf{b}} & :=\bigcap\left\{A_{i}^{c}: b_{i}=0\right\} \cap \bigcap\left\{A_{i}: b_{i}=1\right\}, \\
S_{\mathbf{b}} & :=A_{\mathbf{b}} \cup \bigcup\left\{A_{b_{1}, \ldots, b_{i-1}, 0}: b_{i}=1\right\}=\bigcup\left\{A_{\mathbf{b}^{\prime}}: \mathbf{b}^{\prime} \leq_{\operatorname{lex}} \mathbf{b}\right\},
\end{aligned}
$$

where $\leq_{\text {lex }}$ denotes the lexicographical order (which we apply only to two binary sequences of the same length). Next, for $x \in S$ define $\mathbf{b}(x):=\left(b_{1}(x), b_{2}(x), \ldots\right) \in$ $\{0,1\}^{\mathbb{N}}$ by $b_{i}=I_{A_{i}}$ for $i \in \mathbb{N}$. Thus $\mathbf{b}(x)$ is the unique infinite sequence with $x \in A_{\mathbf{b}(x)}$. Finally, we define

$$
f(x):=\mu\left(S_{\mathbf{b}(x)}\right) .
$$

Claim 4.3. The function $f$ is $\mathcal{F}$-measurable.

Proof of Claim. The function $(x, y) \mapsto I_{\leq_{\text {lex }}}(\mathbf{b}(x), \mathbf{b}(y))$ is $\mathcal{F} \otimes \mathcal{F}$-measurable: the pre-image of 0 is

$$
\left.\bigcup_{i \in \mathbb{N} b_{1}, \ldots, b_{i-1}} \bigcup_{b_{1}, \ldots, b_{i-1}, 1} \times A_{b_{1}, \ldots, b_{i-1}, 0}\right) \in \mathcal{F} \otimes \mathcal{F}
$$

Thus $f(x)=\int I_{\leq_{\text {lex }}}(\mathbf{b}(y), \mathbf{b}(x)) \mathrm{d} \mu(y)$ is $\mathcal{F}$-measurable by [2, Corollary 3.3.3].

Claim 4.4. For every $a \in[0,1]$ and every infinite $\mathbf{b}$, both sets $f^{-1}(a)$ and $A_{\mathbf{b}}$ belong to $\mathcal{F}$ and have $\mu$-measure zero.

Proof of Claim. We have $f^{-1}(a) \in \mathcal{F}$ by Claim 4.3 and $A_{\mathbf{b}} \in \mathcal{F}$ because each $A_{i}$ is in $\mathcal{F}$. Each of $f^{-1}(a)$ and $A_{\mathbf{b}}$ is a null set because otherwise, by Claim 4.2 some $A_{i}$ would cut it into two parts of positive measure, which is clearly impossible.

Claim 4.5. Let $|\mathbf{b}|$ denote the length of the sequence $\mathbf{b}$. Then

$$
\lim _{n \rightarrow \infty} \sup _{|\mathbf{b}|=n} \mu\left(A_{\mathbf{b}}\right)=0 .
$$

Proof of Claim. Assume to the contrary that this limsup is $\varepsilon>0$. Then, by König's lemma ([7, Lemma 8.2.1]), there exists an infinite sequence $\mathbf{b}=\left(b_{1}, b_{2}, \ldots\right)$ such that $\mu\left(A_{b_{1}, \ldots, b_{n}}\right) \geq \varepsilon$ for every $n$. (Note that $A_{b_{1}, \ldots, b_{n}} \supseteq A_{b_{1}, \ldots, b_{n+1}}$.) As $A_{\mathbf{b}}=\bigcap_{n=1}^{\infty} A_{b_{1}, \ldots, b_{n}}$, we conclude that $\mu\left(A_{\mathbf{b}}\right) \geq \varepsilon>0$, contradicting Claim 4.4 . 
Claim 4.6. The set $\left\{\mu\left(S_{\mathbf{b}}\right):|\mathbf{b}|\right.$ is finite $\}$ is dense in $[0,1]$.

Proof of Claim. Consider the binary sequences of length $n$. Notice that, for any finite $\mathbf{b}$,

$$
\mu\left(S_{\mathbf{b}}\right)=\sum_{\mathbf{b}^{\prime} \leq \operatorname{lex}_{\mathbf{b}} \mathbf{b}} \mu\left(A_{\mathbf{b}^{\prime}}\right),
$$

$\mu\left(S_{1,1, \ldots, 1}\right)=1$, and that $\mu\left(S_{0,0, \ldots, 0}\right)=\mu\left(A_{0,0, \ldots 0}\right)$ (which tends to 0 by Claim 4.4). Let $\mathbf{b}^{\prime} \leq_{\text {lex }} \mathbf{b}^{\prime \prime}$ be two sequences of length $n$ which are consecutive in $\leq_{\text {lex }}$. Then

$$
\mu\left(S_{\mathbf{b}^{\prime \prime}}\right)-\mu\left(S_{\mathbf{b}^{\prime}}\right)=\mu\left(A_{\mathbf{b}^{\prime \prime}}\right) \leq \sup _{|\mathbf{b}|=n} \mu\left(A_{\mathbf{b}}\right) .
$$

Combining this with Claim 4.5 gives the statement.

Claim 4.7. The function $f$ is measure-preserving.

Proof of Claim. Claim 4.6 implies that the intervals $\left[0, \mu\left(S_{\mathbf{b}}\right)\right]$, where $\mathbf{b}$ runs over finite binary sequences, generate the Borel $\sigma$-algebra. Thus it is enough to show that for every finite $\mathbf{b}$ we have $\mu\left(f^{-1}([0, a])\right)=a$, where $a:=\mu\left(S_{\mathbf{b}}\right)$. The latter identity follows from the fact that the symmetric difference of $S_{\mathbf{b}}$ and $f^{-1}([0, a])$ is a subset of $f^{-1}(a)$ and therefore has measure zero by Claim 4.4 .

The set $Y:=\left\{(x, y) \in S^{2}: x \triangleleft y, f(x)>f(y)\right\}$ is a subset of $\bigcup_{i=1}^{\infty}\left(A_{i} \times A_{i}^{c}\right) \in$ $\mathcal{F} \otimes \mathcal{F}$. But the latter set has $\mu \otimes \mu$-measure zero by the definition of $A_{i}$ 's. Thus $Y$ also has measure zero. Next, consider the set $Y_{0}:=\left\{(x, y) \in S^{2}: f(x)=f(y)\right\}$. Since $f$ is $\mathcal{F}$-measurable, we have $Y_{0} \in \mathcal{F} \otimes \mathcal{F}$. Every slice of $Y_{0}$ has measure zero by Claim 4.4. By Fubini's theorem, $Y_{0}$ has itself measure zero. We conclude that $f$ is an inclusion.

Finally, take an arbitrary $A \in \mathcal{F}$ with $\mu_{\triangleleft}\left(A \times A^{c}\right)=0$. For every $i \in \mathbb{N}$ there is a set $A_{n_{i}} \in \mathcal{T}$ such that $\mu\left(A_{n_{i}} \triangle A\right)<2^{-i}$. Since $A_{n_{i}} \triangle f^{-1}(X) \subseteq f^{-1}(Y)$, where $X$ is some finite union of intervals and $Y$ is the set of their endpoints, we have by Claims 4.3 and 4.4 that $A_{n_{i}}$ is $\left(f^{-1}(\mathcal{B})\right)_{\mu}$-measurable. This implies that $A$ is $\left(f^{-1}(\mathcal{B})\right)_{\mu}$-measurable: indeed, for

$$
A^{\prime}:=\limsup _{i \rightarrow \infty} A_{n_{i}}=\bigcap_{k=1}^{\infty} \bigcup_{j=k}^{\infty} A_{n_{j}} \in\left(f^{-1}(\mathcal{B})\right)_{\mu}
$$

we have $\mu\left(A^{\prime} \triangle A\right)=0$. This finishes the proof of Theorem 4.1 (and Theorem 1.10).

\section{Proof of Theorem 1.9}

As in Theorem 1.10, we can assume that $\mathcal{F}$ is separable. Apply Theorem 4.1 to $(S, \mathcal{F}, \mu, \triangleleft)$ to obtain an inclusion $f: S \rightarrow[0,1]$. As we will see later, the same $f$ will work in Theorem 1.9. (Thus, rather interestingly, $f$ can be chosen independently of $W$ in Theorem 1.9 if $\mathcal{F}$ is separable.) Let

$$
\mathcal{A}:=\left(f^{-1}(\mathcal{B})\right)_{\mu} .
$$

Since $f$ is $\mathcal{F}$-measurable, we have that $\mathcal{A} \subseteq \mathcal{F}_{\mu}$. 
We would like to apply Lemma 3.3. In order to do so, we have to verify first that $W \sim E(W \mid \mathcal{A} \otimes \mathcal{A})$. (Note that $E(W \mid \mathcal{A} \otimes \mathcal{A}) \sim E\left(W \mid f^{-1}(\mathcal{B}) \otimes f^{-1}(\mathcal{B})\right)$.)

Claim 5.1. For every $y$, the slice function $W_{y}$ is $\mathcal{A}$-measurable.

Proof of Claim. Fix any $a \in[0,1]$. For every $y \in S$, the set

$$
A:=W_{y}^{-1}((a, 1])=\{x \in S: W(x, y)>a\}
$$

is in $\mathcal{F}$. Since $W$ is a strict kernel, we have $\mu_{\triangleleft}\left(A^{c} \times A\right)=0$ for every $y$. By the second part of Theorem 4.1 $A \in \mathcal{F}$ belongs in fact to $\mathcal{A}$. Since intervals $(a, 1]$ generate the Borel $\sigma$-algebra, the claim follows.

The functions $W$ and $\mathbb{E}\left(W \mid \mathcal{A} \otimes \mathcal{F}_{\mu}\right)$ are both $\mathcal{F} \otimes \mathcal{F}$-measurable. (Note that $\mathcal{F} \bar{\otimes} \mathcal{F}=\mathcal{F}_{\mu} \bar{\otimes} \mathcal{F}_{\mu}$.) Also, their $y$-slices are equivalent for a.e. $y$ by Lemma 3.5 and Claim [5.1. By Fubini's theorem, the subset of $S^{2}$ where these two functions differ has $\mu \otimes \mu$-measure zero. In other words, $W$ is $\mathcal{A} \bar{\otimes} \mathcal{F}$-measurable and, by symmetry, $\mathcal{F} \bar{\otimes} \mathcal{A}$-measurable.

Claim 5.2.

$$
W \sim \mathbb{E}(W \mid \mathcal{A} \otimes \mathcal{A})
$$

Proof of Claim. We follow the argument of Borgs, Chayes, and Lovász 3, Section 3.3.5]. Let $\widetilde{W} \in \mathbb{E}(W \mid \mathcal{A} \otimes \mathcal{A})$. It is enough to prove that for every $A, B \in \mathcal{F}$,

$$
\int_{A \times B} W=\int_{A \times B} \widetilde{W}
$$

Take any $g_{A} \in \mathbb{E}\left(I_{A} \mid \mathcal{A}\right)$ and $g_{B} \in \mathbb{E}\left(I_{B} \mid \mathcal{A}\right)$. Define

$$
\begin{aligned}
U_{A}(y) & :=\int_{A} W(x, y) \mathrm{d} \mu(x)=\int W(x, y) I_{A}(x) \mathrm{d} \mu(x), \\
V_{B}(x) & :=\int W(x, y) g_{B}(y) \mathrm{d} \mu(y) .
\end{aligned}
$$

Clearly, $g_{A}$ is $\mathcal{A}$-measurable. Since $W$ is $\mathcal{F} \bar{\otimes} \mathcal{A}$-measurable (as it was noted after Claim [5.1), $U_{A}$ is $\mathcal{A}$-measurable by Fubini's theorem. Similarly, $V_{B}$ is also $\mathcal{A}$ measurable. Repeatedly using Fubini's theorem and (8), we get

$$
\begin{aligned}
\int_{A \times B} W(x, y) \mathrm{d} \mu(x) \mathrm{d} \mu(y) & =\int U_{A}(y) I_{B}(y) \mathrm{d} \mu(y) \\
=\int U_{A}(y) g_{B}(y) \mathrm{d} \mu(y) & =\int W(x, y) I_{A}(x) g_{B}(y) \mathrm{d} \mu(x) \mathrm{d} \mu(y) \\
=\int V_{B}(x) I_{A}(x) \mathrm{d} \mu(x) & =\int V_{B}(x) g_{A}(x) \mathrm{d} \mu(x) \\
=\int W(x, y) g_{A}(x) g_{B}(y) \mathrm{d} \mu(x) \mathrm{d} \mu(y) & =\int \widetilde{W}(x, y) g_{A}(x) g_{B}(y) \mathrm{d} \mu(x) \mathrm{d} \mu(y) .
\end{aligned}
$$

Observe that $g_{A}(x) g_{B}(y)$ is a conditional expectation of $I_{A}(x) I_{B}(y)$ with respect to $\mathcal{A} \otimes \mathcal{A}$ while $\widetilde{W}$ is measurable in this $\sigma$-algebra. Thus we can replace $g_{A}(x) g_{B}(y)$ by $I_{A}(x) I_{B}(y)$ in the last integral, obtaining $\int_{A \times B} \widetilde{W}$ as desired. 
Thus all assumptions of Lemma 3.3 are satisfied and we obtain that $W \sim U^{f}$ for some almost kernel $U$ on $([0,1], \mathcal{B}, \lambda,<)$. By Lemma 3.1] we can change $U$ on a null set so that $([0,1], \mathcal{B}, \lambda,<, U)$ is a kernel. Clearly, the equivalence $W \sim U^{f}$ is not affected by this. This finishes the proof of Theorem 1.9

\section{A finite Szemerédi-Type Regularity Lemma for posets}

In this section we prove a Szemerédi-type Regularity Lemma for posets, Theorem 6.1] (See Prömel, Steger, and Taraz [16] and Patel [15] for other versions.) We then show in Section 7 that this result can be used to answer Janson's question.

Suppose that $(P, \prec)$ is a poset. For two disjoint sets $X, Y \subseteq P$ we write $X \nprec Y$ if there are no $x \in X$ and $y \in Y$ such that $x \prec y$. An (ordered) partition $\mathcal{P}=$ $\left(V_{1}, \ldots, V_{k}\right)$ of the ground set $P$ is a poset partition if

$$
V_{i} \nprec V_{j}, \quad \text { for all } 1 \leq j<i \leq k .
$$

In other words, every $\prec$-relation that involves vertices from two different parts goes "forward". We refer to members of $\mathcal{P}$ as clusters. Let us say that $\mathcal{R}$ is a poset refinement of $\mathcal{P}$ if $\mathcal{R}$ is a poset partition that refines $\mathcal{P}$ (that is, for each $X \in \mathcal{R}$ there exists $Y \in \mathcal{P}$ such that $X \subseteq Y)$. The restriction of $\mathcal{P}$ to $X \subseteq P$ is $\left.\mathcal{P}\right|_{X}=\left(V_{1} \cap X, \ldots, V_{k} \cap X\right)$. (For notational convenience, we allow empty parts.)

Let $G=G_{P, \prec}$ be an (undirected) graph on the vertex set $P$ with edge set

$$
E(G):=\{\{x, y\}: x \prec y \text { or } y \prec x\} .
$$

Clearly, if we know $G$ and a poset partition $\mathcal{P}$, then we can reconstruct $\prec$ except for pairs lying inside a part. The main idea behind our Regularity Lemma is to find a poset partition of $P$ that is regular with respect to $G$.

The following definitions apply to $A, B \subseteq P$. The density of the pair $(A, B)$ is

$$
d(A, B):=\frac{e(A, B)}{|A||B|}:=\frac{|\{(x, y) \in A \times B: x \prec y\}|}{|A||B|}, \quad \text { if } A, B \neq \emptyset,
$$

and $d(A, B):=0$ otherwise. The pair $(A, B)$ is called $\varepsilon$-regular if $\mid d(A, B)-$ $d(X, Y) \mid<\varepsilon$ for each $X \subseteq A$ and $Y \subseteq B$ with $|X| \geq \varepsilon|A|$ and $|Y| \geq \varepsilon|B|$. When we apply the definition of $\varepsilon$-regularity to $(A, B)$, it will always be the case that $B \nprec A$ (and we obtain the standard graph definition). Also, let

$$
q(A, B):=\frac{|A||B|}{|P|^{2}} d^{2}(A, B) .
$$

For disjoint sets $V_{1}, \ldots, V_{k}, U_{1}, \ldots, U_{m} \subseteq P$, we define

$$
\begin{aligned}
q\left(\left(V_{1}, \ldots, V_{k}\right),\left(U_{1}, \ldots, U_{m}\right)\right) & :=\sum_{i=1}^{k} \sum_{j=1}^{m} q\left(V_{i}, U_{j}\right), \\
q\left(\left(V_{1}, \ldots, V_{k}\right)\right) & :=\sum_{i<j} q\left(V_{i}, V_{j}\right) .
\end{aligned}
$$

The function $q$ is called the index and is crucial in the proof of the original Regularity Lemma. Also, let $\mathbb{I}_{\varepsilon}\left(\left(V_{1}, \ldots, V_{k}\right)\right)$ be the set of pairs $(i, j)$ such that $i<j$ and $\left(V_{i}, V_{j}\right)$ is not $\varepsilon$-regular. 
The sizes of the clusters in our Regularity Lemma can vary vastly (at least in our proof). This is why our next definition is slightly different from the standard one. A poset partition $\mathcal{P}=\left(V_{1}, \ldots, V_{k}\right)$ of $P$ is $\varepsilon$-regular if each $\left|V_{i}\right| \leq \max (\varepsilon|P|, 1)$ and

$$
\sum_{(i, j) \in \mathbb{I}_{\varepsilon}(\mathcal{P})}\left|V_{i}\right|\left|V_{j}\right| \leq \varepsilon\left(\begin{array}{c}
|P| \\
2
\end{array}\right) .
$$

Theorem 6.1 (Regularity Lemma for posets). For each $\varepsilon>0$ there exists a number $M$ such that the following holds. For each poset $(P, \prec)$ with a poset partition $\mathcal{P}$ such that $|\mathcal{P}| \leq 1 / \varepsilon$, there exists a poset refinement $\mathcal{R}$ of $\mathcal{P}$ which is $\varepsilon$-regular and has at most $M$ parts.

Remark 6.2. It is important for our later application in Section 7 that there is no garbage cluster in our partition.

We prove Theorem 6.1 by following Szemerédi's original proof of the Regularity Lemma for graphs [20] (a more accessible reference is for example [7, Section 7.4]). The basic idea is that if a current partition $\mathcal{P}$ is not $\varepsilon$-regular, then we can refine it so that $q(\mathcal{P})$ increases by at least $\delta$, where $\delta>0$ depends on $\varepsilon$ only. Since $q$ is always between 0 and $1 / 2$, we reach an $\varepsilon$-regular partition in at most $1 /(2 \delta)$ refinements. The following index increment lemma estimates by how much we can increase $q$ by subdividing one irregular pair $(A, B)$.

Lemma 6.3. Suppose that $(P, \prec)$ is a poset and $A, B \subseteq P$ are disjoint non-empty sets. If $B \nprec A$ and $(A, B)$ is not $\varepsilon$-regular, then there are partitions $A=Z_{1} \cup Z_{2}$ and $B=Z_{3} \cup Z_{4}$ such that $Z_{2} \nprec Z_{1}, Z_{4} \nprec Z_{3}$, and

$$
q\left(\left(Z_{1}, Z_{2}\right),\left(Z_{3}, Z_{4}\right)\right) \geq q(A, B)+\varepsilon^{4} \frac{|A||B|}{n^{2}} .
$$

Proof. Let $d:=d(A, B)$. Consider a witness of irregularity $(X, Y)$ of the pair $(A, B)$. Assume without loss of generality that $d(X, Y) \geq d+\varepsilon$.

Iteratively, repeat the following as long as possible: replace some $x \in X$ by some $y \in A \backslash X$ with $y \prec x$. Clearly, this operation preserves the size of $X$ and cannot decrease $d(X, Y)$. Also, we have to stop at some point. Let $Z_{1}$ be the final $X$ and let $Z_{2}:=A \backslash Z_{1}$.

Similarly, replace $Y \subseteq B$ by an up-closed subset $Z_{4} \subseteq B$ such that $\left|Z_{4}\right|=|Y|$ and $d\left(Z_{1}, Z_{4}\right) \geq d\left(Z_{1}, Y\right) \geq d+\varepsilon$. Let $Z_{3}:=B \backslash Z_{4}$. Of course, we have that $Z_{2} \nprec Z_{1}$ and $Z_{4} \nprec Z_{3}$.

Note that $\left(Z_{1}, Z_{4}\right)$ demonstrates that the pair $(A, B)$ is not $\varepsilon$-regular. Since $B \nprec A$, such density statements also hold with respect to the (undirected) graph $G_{P, \prec}$ that was defined in (16). Thus the standard index estimates from graph theory apply here. In particular, the proof of Lemma 7.4.3 in [7] shows that (17) holds whenever $\left(Z_{1}, Z_{4}\right)$ is a witness of $\varepsilon$-irregularity.

Proof of Theorem 6.1. Let $s:=\left\lceil 2 / \varepsilon^{5}\right\rceil, k_{0}:=\lceil 2 / \varepsilon\rceil$, and inductively for $t=$ $0, \ldots, s-1$, let $k_{t+1}:=k_{t} 2^{k_{t}-1}$. We claim that $M=k_{s}$ suffices.

Suppose that $n:=|\mathcal{P}|>1 / \varepsilon$ for otherwise we can let $\mathcal{R}$ be a partition into singletons.

Initially, let $\mathcal{R}_{0}$ be an arbitrary poset refinement of $\mathcal{P}$ such that $\left|\mathcal{R}_{0}\right| \leq k_{0}$ and each part has at most $\varepsilon n$ vertices.

Iteratively, for $t=0,1, \ldots$, we repeat the following procedure. Let $\mathcal{R}_{t}=$ $\left(V_{1}, \ldots, V_{k}\right)$. If $\mathcal{R}_{t}$ is $\varepsilon$-regular, then we stop and output $\mathcal{R}_{t}$; so suppose otherwise. 
Let $\mathcal{R}^{\prime}:=\mathcal{R}_{t}$. We modify $\mathcal{R}^{\prime}$ by using another (embedded) iterative procedure. Namely, in turn for each $(i, j) \in \mathbb{I}_{\varepsilon}\left(\mathcal{R}_{t}\right)$, we take the partitions $V_{i}=Z_{1 i j} \cup Z_{2 i j}$ and $V_{j}=Z_{3 i j} \cup Z_{4 i j}$ returned by Lemma 6.3 and replace every $X \in \mathcal{R}^{\prime}$ by $X \cap Z_{1 i j}, \ldots, X \cap Z_{4 i j}$, with these four parts coming in the specified order. Clearly, $\mathcal{R}^{\prime}$ is still a poset partition. Once we have processed all elements of $\mathbb{I}_{\varepsilon}\left(\mathcal{R}_{t}\right)$, we let $\mathcal{R}_{t+1}:=\mathcal{R}^{\prime}$.

In order to estimate how $q$ changes, let us write

$$
q\left(\mathcal{R}_{t+1}\right)-q\left(\mathcal{R}_{t}\right) \geq \sum_{1 \leq i<j \leq k}\left(q\left(\left.\mathcal{R}_{t+1}\right|_{V_{i}},\left.\mathcal{R}_{t+1}\right|_{V_{j}}\right)-q\left(V_{i}, V_{j}\right)\right)
$$

where the inequality comes from discarding the sum $\sum_{i=1}^{k} q\left(\left.\mathcal{R}_{t+1}\right|_{V_{i}}\right) \geq 0$. We can estimate each summand corresponding to $(i, j) \in \mathbb{I}_{\varepsilon}\left(\mathcal{R}_{t}\right)$ by passing from $q\left(V_{i}, V_{j}\right)$ first to $q\left(\left(Z_{1 i j}, Z_{2 i j}\right),\left(Z_{3 i j}, Z_{4 i j}\right)\right)$ and then to $q\left(\left.\mathcal{R}_{t+1}\right|_{V_{i}},\left.\mathcal{R}_{t+1}\right|_{V_{j}}\right)$. The first step increases $q$ as specified by Lemma 6.3. The second step has non-negative effect by [7, Lemma 7.4.2]. Each other term in the right-hand side of (18) is non-negative, again by [7, Lemma 7.4.2]. Since $\mathcal{R}_{t}$ is not $\varepsilon$-regular, we conclude that

$$
q\left(\mathcal{R}_{t+1}\right)-q\left(\mathcal{R}_{t}\right) \geq \frac{\varepsilon^{4}}{n^{2}} \sum_{(i, j) \in \mathbb{I}_{\varepsilon}\left(\mathcal{R}_{t}\right)}\left|V_{i}\right|\left|V_{j}\right|>\frac{\varepsilon^{4}}{n^{2}} \varepsilon\left(\begin{array}{l}
n \\
2
\end{array}\right) \geq \frac{\varepsilon^{5}}{4} .
$$

Trivially, $0 \leq q(\mathcal{P}) \leq 1 / 2$ for any partition $\mathcal{P}$. By (19), we repeat the iteration procedure at most $s$ times before we reach an $\varepsilon$-regular poset partition. As each part of $\mathcal{R}^{t}$ is split into at most $2^{\left|\mathcal{R}_{t}\right|-1}$ parts, we have that $\left|\mathcal{R}^{t+1}\right| \leq\left|\mathcal{R}_{t}\right| 2^{\left|\mathcal{R}_{t}\right|-1}$. Thus the final partition has at most $M$ parts, as required.

If we do not know $\prec$ but know an $\varepsilon$-regular partition $\mathcal{R}=\left(V_{1}, \ldots, V_{k}\right)$ and the densities between all pairs of parts, then we can still derive various information about the poset $P$. For example, given two subsets $S, T \subseteq P$, one would expect to see approximately

$$
e^{\prime}(S, T):=\sum_{i<j} d\left(V_{i}, V_{j}\right)\left|V_{i} \cap S\right|\left|V_{j} \cap T\right|
$$

directed arcs from $S$ to $T$. Indeed, this is the case for posets.

Lemma 6.4. Given the above assumptions, we have

$$
\left|e(S, T)-e^{\prime}(S, T)\right| \leq 3 \varepsilon\left(\begin{array}{c}
|P| \\
2
\end{array}\right) .
$$

Proof. Let $n:=|P|$. Assuming the worst-case scenario, the edges inside a part or inside a non- $\varepsilon$-regular pair contribute at most $\varepsilon\left(\begin{array}{l}n \\ 2\end{array}\right)+\varepsilon\left(\begin{array}{c}n \\ 2\end{array}\right)$ to the left-hand side of (20). For every $\varepsilon$-regular pair $\left(V_{i}, V_{j}\right)$ with $i<j$, we have

$$
\left|e\left(V_{i} \cap S, V_{j} \cap T\right)-d\left(V_{i}, V_{j}\right)\right| V_{i} \cap S|| V_{j} \cap T|| \leq \varepsilon\left|V_{i}\right|\left|V_{j}\right| .
$$

Indeed, if $\left|V_{i} \cap S\right|\left|V_{j} \cap T\right| \leq \varepsilon\left|V_{i}\right|\left|V_{j}\right|$, then we are trivially done; otherwise both $S$ and $T$ take more than $\varepsilon$-proportion of respectively $V_{i}$ and $V_{j}$ and the bound follows by the $\varepsilon$-regularity of $\left(V_{i}, V_{j}\right)$. Thus the aggregate contribution of $\varepsilon$-regular pairs to (20) is at most $\varepsilon\left(\begin{array}{l}n \\ 2\end{array}\right)$. 


\section{An alternative Proof of Theorem 1.7}

Let $\left\{\left(P_{n}, \prec_{n}\right)\right\}_{n \in \mathbb{N}}$ be a convergent sequence of posets. We have to construct a kernel $([0,1], \mathcal{B}, \lambda,<, U)$ such that for every poset $P$ we have

$$
t(P, U)=\lim _{n \rightarrow \infty} t\left(P, P_{n}\right) .
$$

We construct $U$ following closely the analogous construction of Lovász and Szegedy [13, Theorem 2.2] (see also [14, Theorem 5.1]). In brief, the proof proceeds by finding a $\frac{1}{k}$-regular partition $\mathcal{P}_{n, k}$ of $P_{n}$ with the number of parts bounded by a function of $k$ only. Then we construct a step-function $W_{n, k}:[0,1]^{2} \rightarrow[0,1]$ that encodes the part ratios and densities of $\mathcal{P}_{k, n}$. Since the "complexity" of $W_{n, k}$ is bounded by a function of $k$, a diagonalisation process gives a subsequence $\left\{P_{n_{i}}\right\}_{i \in \mathbb{N}}$ such that, for every $k$, we have $W_{n, k} \rightarrow U_{k}$ a.e. for some $U_{k}:[0,1]^{2} \rightarrow[0,1]$. Additionally, when we choose our partitions $\mathcal{P}_{k, n}$, we can assume that they are nested for each $n$. This allows us to write $U_{k-1}$ as a conditional expectation of $U_{k}$ and conclude that $\left\{U_{k}\right\}_{k \in \mathbb{N}}$ converges to some $U$ a.e. Finally, we need to apply Lemma 3.1 to tranform an almost kernel $U$ into a kernel.

Let us give more details. Let $m_{1}=1$ and inductively for $k=2,3, \ldots$ let $m_{k}$ be sufficiently large such that every poset partition with at most $m_{k-1}$ parts admits a $\frac{1}{k}$-regular poset refinement with $m_{k}$ parts. Such a number exists by Theorem 6.1 . (Recall that we allow empty parts.) For each $n \in \mathbb{N}$, let $\mathcal{P}_{n, 1}:=\left(P_{n}\right)$ be the trivial partition and then inductively for $k=2,3, \ldots$ let

$$
\mathcal{P}_{n, k}=\left(V_{n, k, 1}, \ldots, V_{n, k, m_{k}}\right)
$$

be a $\frac{1}{k}$-regular poset partition of $\left(P_{n}, \prec_{n}\right)$ that refines $\mathcal{P}_{n, k-1}$. This nestedness allows us for each $n$, to choose a total ordering $\prec_{n}^{\prime}$ of $\left(P_{n}, \prec_{n}\right)$ which is compatible with every poset partition $\mathcal{P}_{n, k}$ (that is, $V_{n, k, i} \nprec_{n}^{\prime} V_{n, k, j}$ whenever $i>j$ ). By relabelling, let us assume that $P_{n}=\left\{1, \ldots,\left|P_{n}\right|\right\}$ and $\prec_{n}^{\prime}$ is the standard order.

Already at this point, it makes sense to start operating with functions. Let $W_{n}:[0,1]^{2} \rightarrow\{0,1\}$ be the step-function that encodes the $\prec_{n}$-relation in the obvious way: $W_{n}$ is constant on $\left[\frac{i-1}{v}, \frac{i}{v}\right) \times\left[\frac{j-1}{v}, \frac{j}{v}\right)$, where $v:=\left|P_{n}\right|$, and assumes value 1 there if and only if $i \prec_{n} j$. It is easy to see that

$$
t\left(P, P_{n}\right)=t\left(P, W_{n}\right), \quad \text { for every poset } P
$$

where we view $W_{n}$ as a kernel on $([0,1], \mathcal{B}, \lambda,<)$.

Let $\mathcal{P}_{n, k}^{\prime}=\left(V_{n, k, 1}^{\prime}, \ldots, V_{n, k, m_{k}}^{\prime}\right)$ be the partition of $[0,1]$ into consecutive intervals corresponding to (22). (Thus, for example, $\lambda\left(V_{n, k, i}^{\prime}\right)=\left|V_{n, k, i}\right| /\left|P_{n}\right|$.) Let $W_{n, k}$ be the step-function on $\mathcal{P}_{n, k}^{\prime} \times \mathcal{P}_{n, k}^{\prime}$, whose steps correspond to the parts of $\mathcal{P}_{n, k}$ and whose values correspond to densities between parts. We can write this more compactly as

$$
W_{n, k} \sim \mathbb{E}\left(W_{n} \mid \sigma\left(\mathcal{P}_{n, k}^{\prime}\right)\right),
$$

a conditional expectation of $W_{n}$ with respect to the (finite) $\sigma$-algebra generated by $\mathcal{P}_{n, k}^{\prime}$. Since $\sigma\left(\mathcal{P}_{n, 1}^{\prime}\right) \subseteq \sigma\left(\mathcal{P}_{n, 2}^{\prime}\right) \subseteq \ldots$, we have

$$
W_{n, k} \sim \mathbb{E}\left(W_{n, k+1} \mid \sigma\left(\mathcal{P}_{n, k}^{\prime}\right)\right), \quad k \geq 1,
$$

which translates into the combinatorially obvious fact that the densities of $\mathcal{P}_{n, k}$ can be obtained by averaging over the densities in the finer partition $\mathcal{P}_{n, k+1}$.

Since each $W_{n, k}$ can be described by specifying part sizes and densities (which involves at most $m_{k}+\left(\begin{array}{c}m_{k} \\ 2\end{array}\right)$ reals in $\left.[0,1]\right)$, the standard diagonalisation process 
gives a subsequence $\left\{n_{i}\right\}_{i \in \mathbb{N}}$ such that these parameters converge for every $k$. Thus $W_{n_{i}, k} \rightarrow U_{k}$ a.e. for some step-function $U_{k}$ with $m_{k}$ steps that are intervals and are ordered as $\mathcal{P}_{k}^{\prime}=\left(V_{k, 1}^{\prime}, \ldots, V_{k, m_{k}}^{\prime}\right)$. Since $\left\{P_{n}\right\}_{n \in \mathbb{N}}$ is convergent, passing to a subsequence does not affect (21); thus we can assume that $\left\{W_{n, k}\right\}_{n \in \mathbb{N}}$ itself a.e. converges to $U_{k}$. Clearly, $\sigma\left(\mathcal{P}_{1}^{\prime}\right) \subseteq \sigma\left(\mathcal{P}_{2}^{\prime}\right) \subseteq \ldots$ a.e. and

$$
U_{k} \sim \mathbb{E}\left(U_{k+1} \mid \sigma\left(\mathcal{P}_{k}^{\prime}\right)\right) .
$$

Thus, by the Martingale Convergence Theorem (see e.g. [2, Theorem 10.3.3]), $U_{k} \rightarrow$ $U$ a.e. for some $U:[0,1]^{2} \rightarrow[0,1]$.

The obtained function $U$, as the a.e. pointwise limit of Borel functions, is Borel a.e. Clearly, the kernel axioms hold for $([0,1], \mathcal{B}, \lambda, U)$ for all inputs that do not require the evaluation of $U$ on a point of

$$
X:=\left\{(x, y) \in[0,1]^{2}: U_{k}(x, y) \nrightarrow \nrightarrow U(x, y) \text { or } \exists k W_{n, k}(x, y) \nrightarrow U_{k}(x, y)\right\},
$$

the set where some convergence fails. Since $X$ has measure zero, $U$ is an almost kernel. By applying Lemma 3.1, we can assume that $U$ is a kernel.

It remains to show that (21) holds. The cut-norm of a bounded measurable function $W:[0,1]^{2} \rightarrow \mathbb{R}$ is defined by

$$
\|W\|_{\square}=\sup _{S, T \in \mathcal{B}}\left|\int_{S \times T} W(x, y) \mathrm{d} \lambda(x) \mathrm{d} \lambda(y)\right| .
$$

Claim 7.1. $\left\|W_{n}-W_{n, k}\right\|_{\square} \leq \frac{5}{2 k}$ for any $k, n \in \mathbb{N}$.

Proof of Claim. Let $W:=W_{n}-W_{n, k}$. Assume that $v:=\left|P_{n}\right|>k$ for otherwise there is nothing to do as $W=0$.

Observe that, up to an additive error $\frac{1}{v}$, it is enough to consider those $S$ and $T$ in (23) that are unions of intervals $V_{i}:=\left[\frac{i-1}{v}, \frac{i}{v}\right)$ for $i \in[v]$. Indeed, fix any $S, T \in \mathcal{B}$ with, say, $\int_{S \times T} W \geq 0$ and take $i \in[v]$ one by one. If we modify $S$ and $T$ inside $V_{i}$, then the integral of $W$ over

$$
\left(\left(V_{i} \times V_{i}^{c}\right) \cup\left(V_{i}^{c} \times V_{i}\right)\right) \cap(S \times T)
$$

is a linear function of $\lambda\left(V_{i} \cap S\right)$ and $\lambda\left(V_{i} \cap T\right)$. Thus we can make each of these belong to $\{0,1 / v\}$ without decreasing the above contribution. Updating $S$ and $T$ accordingly, we decrease $\int_{S \times T} W$ by at most $\int_{V_{i} \times V_{i}}|W| \leq 1 / v^{2}$. When we have iteratively processed all $i \in[v]$, both $S$ and $T$ have the desired form.

Thus, by (20), we obtain the required:

$$
\|W\|_{\square} \leq \frac{3}{k}\left(\begin{array}{l}
v \\
2
\end{array}\right) \frac{1}{v^{2}}+\frac{1}{v} \leq \frac{3}{2 k}+\frac{1}{k}=\frac{5}{2 k} .
$$

Now, we are ready to verify (21). Take any poset $(P, \prec)$ and $\varepsilon>0$. Let $m:=$ $e\left(G_{P, \prec}\right)$ be the number of pairs in $\prec$.

Since we deal with bounded measurable functions, all convergences also hold in the $\ell_{1}$-space on $\left([0,1]^{2}, \mathcal{B}, \lambda\right)$ by [2, Theorem 2.2.3]. Thus there is $k \geq \frac{15 m}{2 \varepsilon}$ such that $\left\|U-U_{k}\right\|_{1} \leq \frac{\varepsilon}{3 m}$ and, fixing this $k$, there is $n_{0}$ such that $\left\|U_{k}-W_{n, k}\right\|_{1} \leq \frac{\varepsilon}{3 m}$ for all $n \geq n_{0}$. Clearly, $\|f\|_{\square} \leq\|f\|_{1}$ for any integrable $f$. Thus, by the Triangle 
Inequality and Claim 7.1, we have that for all $n \geq n_{0}$

$$
\begin{aligned}
\left\|U-W_{n}\right\|_{\square} & \leq\left\|U-U_{k}\right\|_{\square}+\left\|U_{k}-W_{n, k}\right\|_{\square}+\left\|W_{n, k}-W_{n}\right\|_{\square} \\
& \leq\left\|U-U_{k}\right\|_{1}+\left\|U_{k}-W_{n, k}\right\|_{1}+\frac{5}{2 k} \leq \frac{\varepsilon}{m} .
\end{aligned}
$$

By [11, Lemma 6.4], we have that $\left|t(P, U)-t\left(P, W_{n}\right)\right| \leq m\left\|U-W_{n}\right\|_{\square} \leq \varepsilon$. Since $\varepsilon$ and $P$ were arbitrary, (21) follows.

Summarising, $([0,1], \mathcal{B}, \lambda,<, U)$ is a kernel that establishes Theorem 1.7 .

Remark 7.2. An alternative way to proving that the densities of $F$ in $W_{n}$ and $W_{n, k}$ are close is to adopt the Counting Lemma (see e.g. [18, Theorem 5]) to our settings. We do not see any principal difficulties here but we expect that the error term would be larger.

Remark 7.3. In the above proof it is not generally true that $\left\|W_{n}-W_{n, k}\right\|_{1}$ is small for sufficiently large $k$ : for example, $W_{n, k}$ may be strictly between 0 and 1 on a set of positive measure (while $W_{n}$ is always $\{0,1\}$-valued).

\section{Concluding REMARKS}

There are two natural ways to extend the definition of convergence to the case when the poset orders do not tend to infinity. One is to just use (1). Another, adopted by Janson [11, Definition 3.2], is to say that $\left\{P_{n}\right\}_{n \in \mathbb{N}}$ with $\left|P_{n}\right| \not \rightarrow \infty$ is convergent if the sequence is eventually constant (up to isomorphism). The choice of which one to use (or none) is more a matter of convenience. For example, this choice may depend on whether we want the "limits" of $(P, P, \ldots)$ and $\left(P^{(1)}, P^{(2)}, \ldots\right)$ to be the same or not. Here the blow-up $P^{(k)}$ of $P$ is obtained by cloning $k$ times each vertex of $P$; obviously, $t(Q, P)=t\left(Q, P^{(k)}\right)$ for every poset $Q$. Since all results stated in the introduction can be trivially reduced to the case $\left|P_{n}\right| \rightarrow \infty$ by blowing posets up, we decided to use Definition 1.1.

Of course, the assumption that $(S, \mathcal{F}, \mu)$ is atomless is necessary in Theorems 1.9 and 1.10. This assumption can be removed if we are allowed to modify $([0,1], \mathcal{B}, \lambda,<)$ by shifting positive measure to a some countable subset $X \subseteq[0,1]$, where $X$ depends on $(S, \mathcal{F}, \mu, \triangleleft)$. However, we believe that the versions presented in the introduction are neater.

We cannot require in Theorems 1.9 and 1.10 that $f$ preserves every relation (i.e. that the set in (7) is empty) as the following example demonstrates. Let $S:=[0,1)$ with the Lebesgue measure $\lambda$ on the Borel $\sigma$-algebra $\mathcal{B}$. Fix an irrational number $\tau$. Let $T: S \rightarrow S$ map $x$ to $x+\tau(\bmod 1)$. If we view $S$ as a circle, then $T$ is an aperiodic rotation. Define $x \triangleleft y$ if there is $k \in \mathbb{N}$ with $y=T^{k}(x)$. The constructed relation $\triangleleft$ is a Borel subset of $S^{2}$ (of measure zero). Let us suppose to the contrary that there is an inclusion $f:(S, \mathcal{B}, \lambda, \triangleleft) \rightarrow([0,1], \mathcal{B}, \lambda,<)$ such that the set in (7) is empty. Let $A:=f^{-1}\left(\left[0, \frac{1}{2}\right]\right)$. Since $A$ is a down-closed set with respect to $<$, we have that $T^{-1}(A) \subseteq A$. Since $T$ is measure-preserving, we conclude that $T^{-1}(A) \sim A$. However, this contradicts the well-known fact (see e.g. [2, Example 10.9.9]) that $T$ is ergodic. Alternatively, let $B:=\bigcap_{k=1}^{\infty} T^{-k}(A)$. Then $B$ is a measurable set such that $T^{-1}(B)=B$ (exactly) and $\mu(B)=1 / 2$ (by $\sigma$-additivity). The same applies to $B^{c}$. By taking density points $x$ and $y$ of $B$ and $B^{c}$ respectively and a sequence of $k$ such that $T^{k}(x) \rightarrow y$, one readily arrives at the desired contradiction. 
Also, the assumption that $W$ is strict in Theorem 1.9 is needed. For example, take $[0,1]^{2}$ with the Lebesgue measure on the Borel sets and let $(x, y) \triangleleft\left(x^{\prime}, y^{\prime}\right)$ if $x<x^{\prime}$. Let, for example, $W\left((x, y),\left(x^{\prime}, y^{\prime}\right)\right)$ be $y^{\prime}$ if $x^{\prime}>x+1 / 2$ and 0 otherwise. It is easy to see that every inclusion of this ordered probability space into the unit interval is a.e. equal to the projection onto the first coordinate. However, $W\left((x, y),\left(x^{\prime}, y^{\prime}\right)\right)$ is essentially non-constant on $\left(x, x^{\prime}\right)$-slices for $x^{\prime}>1 / 2+x$ and thus cannot be equivalent to some pull-back.

\section{ACKNOWLEDGEMENT}

The authors thank the anonymous referee for helpful comments.

\section{REFERENCES}

[1] Tim Austin, On exchangeable random variables and the statistics of large graphs and hypergraphs, Probab. Surv. 5 (2008), 80-145, DOI 10.1214/08-PS124. MR2426176 (2010g:60016)

[2] Vladimir Bogachev, Measure theory. Vol. I, II, Springer-Verlag, Berlin, 2007. MR2267655 (2008g:28002)

[3] Christian Borgs, Jennifer Chayes, and László Lovász, Moments of two-variable functions and the uniqueness of graph limits, Geom. Funct. Anal. 19 (2010), no. 6, 1597-1619, DOI 10.1007/s00039-010-0044-0. MR2594615(2011d:05242)

[4] Christian Borgs, Jennifer Chayes, László Lovász, Vera T. Sós, and Katalin Vesztergombi, Convergent sequences of dense graphs. I. Subgraph frequencies, metric properties and testing, Adv. Math. 219 (2008), no. 6, 1801-1851, DOI 10.1016/j.aim.2008.07.008. MR2455626 (2009m:05161)

[5] Graham Brightwell and Nicholas Georgiou, Continuum limits for classical sequential growth models, Random Structures Algorithms 36 (2010), no. 2, 218-250, DOI 10.1002/rsa.20278. MR2583061 (2011f:60190)

[6] Persi Diaconis and Svante Janson, Graph limits and exchangeable random graphs, Rend. Mat. Appl. (7) 28 (2008), no. 1, 33-61. MR2463439(2010a:60127)

[7] Reinhard Diestel, Graph theory, 4th ed., Graduate Texts in Mathematics, vol. 173, Springer, Heidelberg, 2010. MR2744811 (2011m:05002)

[8] Gábor Elek and Balázs Szegedy, A measure-theoretic approach to the theory of dense hypergraphs, Adv. Math. 231 (2012), no. 3-4, 1731-1772, DOI 10.1016/j.aim.2012.06.022. MR2964622

[9] Carlos Hoppen, Yoshiharu Kohayakawa, Carlos Gustavo Moreira, Balázs Ráth, and Rudini Menezes Sampaio, Limits of permutation sequences, J. Combin. Theory Ser. B 103 (2013), no. 1, 93-113, DOI 10.1016/j.jctb.2012.09.003. MR2995721

[10] Carlos Hoppen, Yoshiharu Kohayakawa, Carlos Gustavo Moreira, and Rudini Menezes Sampaio, Limits of permutation sequences through permutation regularity, E-Print arXiv.org: 1106.1663, 2011.

[11] Svante Janson, Poset limits and exchangeable random posets, Combinatorica 31 (2011), no. 5, 529-563, DOI 10.1007/s00493-011-2591-x. MR2886098

[12] Svante Janson, Limits of interval orders and semiorders, J. Comb. 3 (2012), no. 2, 163-183, DOI 10.4310/JOC.2012.v3.n2.a2. MR2980748

[13] László Lovász and Balázs Szegedy, Limits of dense graph sequences, J. Combin. Theory Ser. B 96 (2006), no. 6, 933-957, DOI 10.1016/j.jctb.2006.05.002. MR2274085 (2007m:05132)

[14] László Lovász and Balázs Szegedy, Szemerédi's lemma for the analyst, Geom. Funct. Anal. 17 (2007), no. 1, 252-270, DOI 10.1007/s00039-007-0599-6. MR2306658 (2008a:05129)

[15] Viresh Patel, Partitions of combinatorial structures, PhD Thesis, London School of Economics, 2009.

[16] Hans Jürgen Prömel, Angelika Steger, and Anusch Taraz, Counting partial orders with a fixed number of comparable pairs, Combin. Probab. Comput. 10 (2001), no. 2, 159-177, DOI 10.1017/S0963548301004503. MR1833068(2002e:06003)

[17] Alexander A. Razborov, Flag algebras, J. Symbolic Logic 72 (2007), no. 4, 1239-1282, DOI 10.2178/jsl/1203350785. MR2371204 (2008j:03040) 
[18] Miklós Simonovits and Vera T. Sós, Szemerédi's partition and quasirandomness, Random Structures Algorithms 2 (1991), no. 1, 1-10, DOI 10.1002/rsa.3240020102. MR.1099576 (92c:05142)

[19] Balázs Szegedy, Gowers norms, regularization and limits of functions on abelian groups, E-Print arxiv.org:1010.6211, 2010.

[20] Endre Szemerédi, Regular partitions of graphs (English, with French summary), Problèmes combinatoires et théorie des graphes (Colloq. Internat. CNRS, Univ. Orsay, Orsay, 1976), Colloq. Internat. CNRS, vol. 260, CNRS, Paris, 1978, pp. 399-401. MR.540024 (81i:05095)

[21] Terence Tao, A correspondence principle between (hyper)graph theory and probability theory, and the (hyper)graph removal lemma, J. Anal. Math. 103 (2007), 1-45, DOI 10.1007/s11854008-0001-0. MR2373263 (2009e:60022)

Mathematics Institute, University of Warwick, Coventry, CV4 7AL, United Kingdom E-mail address: J.Hladky@warwick.ac.uk

Current address: Institute of Mathematics, Academy of Sciences of the Czech Republic, Žitná 25, 110 00, Praha, Czech Republic

E-mail address: honzahladky@gmail.com

Mathematics Institute, University of Warwick, Coventry, CV4 7AL, United Kingdom E-mail address: A.Mathe@warwick.ac.uk

School of Mathematics, Birmingham University, Edgbaston, Birmingham B15 2TT, UNITED KINGDOM

E-mail address: viresh.s.patel@googlemail.com

Current address: School of Mathematical Sciences, Queen Mary, University of London, Mile End Road, London E1 4NS, United Kingdom

Mathematics Institute and DimAP, University of Warwick, Coventry, CV4 7AL, UNITED KINGDOM

URL: http://homepages.warwick.ac.uk/staff/O.Pikhurko/ 